\title{
A Review of Structural MRI and Diffusion Tensor Imaging in Schizotypal Personality Disorder
}

\author{
Erin A. Hazlett • Kim E. Goldstein • Jeanine C. Kolaitis
}

Published online: 18 October 2011

(C) The Author(s). This article is published with open access at Springerlink.com 2011

\begin{abstract}
Individuals with schizotypal personality disorder (SPD) share genetic, phenomenologic, and cognitive abnormalities with people diagnosed with schizophrenia. To date, 15 structural MRI studies of the brain have examined size, and 3 diffusion tensor imaging studies have examined white matter connectivity in SPD. Overall, both types of structural neuroimaging modalities have shown temporal lobe abnormalities similar to those observed in schizophrenia, while frontal lobe regions appear to show more sparing. This intriguing pattern suggests that frontal lobe sparing may suppress psychosis, which is consistent with the idea of a possible neuroprotective factor. In this paper, we review these 18 studies and discuss whether individuals with SPD who both resemble and differ from schizophrenia patients in their phenomenology, share some or all of the structural brain imaging characteristics of schizophrenia. We attempt to group the MRI abnormalities in SPD into three patterns: 1) a spectrum of severityabnormalities are similar to those observed in schizophrenia but not so severe; 2) a spectrum of region-abnormalities affecting some, but not all, brain regions affected in
\end{abstract}

E. A. Hazlett $(\bowtie) \cdot$ J. C. Kolaitis

Mental Illness Research, Education, and Clinical Center (VISN3),

James J. Peters VA Medical Center,

130 West Kingsbridge Road, Room 6A-45,

Bronx, NY 10468, USA

e-mail: erin.hazlett@mssm.edu

E. A. Hazlett $\cdot$ J. C. Kolaitis

Department of Psychiatry, Mount Sinai School of Medicine,

New York, NY, USA

K. E. Goldstein

Department of Psychology, Temple University,

Philadelphia, PA, USA schizophrenia; and 3) a spectrum of compensationabnormalities reflecting greater-than-normal white matter volume, possibly serving as a buffer or compensatory mechanism protecting the individual with SPD from the frank psychosis observed in schizophrenia.

Keywords Schizotypal personality disorder Schizotypy · MRI - Diffusion tensor - Diffusion tensor imaging · Fractional anisotropy DTI - White matter - Gray matter . Morphometry · Schizophrenia · Schizophrenia spectrum . Brain volume $\cdot$ Frontal lobe $\cdot$ Temporal lobe $\cdot$ Superior temporal gyrus $\cdot$ Cingulate $\cdot$ Cingulum

\section{Introduction}

Beginning with the classic work of Kraepelin [1] and Bleuler [2], schizophrenia has been considered to be on a continuum. Twenty years later, Kety et al. [3] described relatives of probands with schizophrenia as having "borderline schizophrenia" or "inadequate personality," which was the basis for the diagnosis of schizotypal personality disorder (SPD). At the same time, the landmark Danish adopted away studies of schizophrenia conducted by Rosenthal and coworkers [4] determined that relatives of patients with schizophrenia displayed deviant psychological functioning, but not all signs of schizophrenia, providing further evidence for a spectrum of schizophrenia-related disorders. Today, SPD is the prototypical schizophrenia spectrum personality disorder. It was first included in the DSM-III and has always been listed under the "odd" cluster of the personality disorders. Individuals with SPD share an extensive array of similarities with schizophrenia patients in terms of phenomenology, genetics, and structural and functional brain imaging [5]. In this paper, we selectively 
review structural MRI studies, including a newer methodology called diffusion tensor imaging (DTI) of individuals with SPD.

SPD is characterized by a pervasive pattern of social and interpersonal deficits and requires five of the nine $D S M$ criteria [6]. Generally, these symptoms include ideas of reference, odd beliefs, unusual perceptual experiences, odd thinking/speech, suspiciousness or paranoid ideation, inappropriate affect, odd behavior, few friends or confidants, and excessive social anxiety that "does not diminish with familiarity and tends to be associated with paranoid fears rather than negative judgments about self' [6, p. 645]. Of note, SPD shares some of these features with chronic schizophrenia, primarily the psychotic-like symptoms (e.g., magical thinking, ideas of reference, and suspiciousness) and the deficit-like symptoms of constricted affect, social isolation, and peculiar appearance and speech. These symptoms have been shown to be discriminated by factor analyses in the independent dimensions of interpersonal deficit, cognitive-disorganization deficit, and paranoid symptoms $[7,8]$. The asociality and cognitive impairments observed in SPD are typically milder than those seen in schizophrenia. In further contrast to schizophrenia, individuals with SPD are not considered to be psychotic and thus, generally have not been exposed to antipsychotic medications or hospitalization. Given the potential effect of antipsychotic medications on brain morphology [e.g., 9], it is useful to examine structural neuroimaging in SPD patients because they are typically drug naïve and have not been chronically hospitalized.

Another important reason to study SPD is to better conceptualize its biological similarities to and differences from schizophrenia using measures such as structural brain imaging. Our review focuses specifically on frontal and temporal lobe findings given that prior MRI studies have primarily compared and contrasted these two regions in SPD and schizophrenia, generally highlighting similar temporal lobe abnormalities in both disorders but different frontal lobe patterns of results (reviewed by Dickey et al. [10] and Siever and Davis [5]). We attempt to group the frontaltemporal MRI and DTI findings into three patterns: 1) a spectrum of severity-abnormalities are similar to those observed in schizophrenia but not so severe; 2 ) a spectrum of region-abnormalities affecting some, but not all, brain regions affected in schizophrenia; and 3) a spectrum of compensation - abnormalities reflecting greater-than-normal white matter volume, possibly serving to protect the individual with SPD from the frank psychosis observed in schizophrenia.

We conducted a PubMed search in August 2011 for English-language articles including the following keywords in various combinations: schizotypal personality disorder, magnetic resonance imaging, MRI, diffusion tensor imaging, and DTI. We found and reviewed 15 MRI studies and 3 DTI studies involving participants with SPD, and Tables 1 and 2 provide a summary of these studies. We have limited our review to studies that included individuals meeting full $D S M-I V$ criteria for SPD and have not included those involving individuals who score high on psychometric scales of psychopathology associated with SPD. However, in general, we wish to acknowledge the importance of studies that begin to use a dimensional approach to examine the traits of SPD, consistent with the recent National Institute of Mental Health Research Domain Criteria strategy.

\section{Structural MRI Studies of Schizotypal Personality Disorder}

\section{Frontal Lobe Findings}

The frontal lobe is one of the major regions to receive attention in the search for the neural substrates of schizophrenia. Several MRI studies have reported volume reductions in schizophrenia (see review by Shenton et al. [11••]). Involvement of the frontal lobe has been related to negative symptoms and cognitive impairments in schizophrenia, including deficits in executive and problem-solving functions and working memory $[12,13]$. Several MRI studies have also examined frontal lobe involvement in SPD, although the findings are more mixed. Yoneyama and colleagues [14] conducted a voxel-based morphometry analysis of MRI data and reported no SPD-control differences in frontal regions. However, a second voxel-based morphometry study demonstrated that schizophrenia patients showed more widespread reductions in the frontal lobe, including bilateral medial frontal, inferior frontal, left middle frontal, and orbitofrontal regions, while individuals with SPD showed limited reductions in only the left inferior frontal region [15]. Raine and colleagues [16] reported reduced prefrontal gray volume in SPD but also showed that group differences were eliminated after controlling for comorbid antisocial behavior. A regionof-interest study conducted by Suzuki et al. [17] reported that SPD patients had larger volumes of the bilateral middle frontal gyrus and smaller volumes of the right straight gyrus compared with healthy controls. In contrast, the schizophrenia patients showed widely reduced volumes of subcomponents of the prefrontal cortex compared with SPD patients and healthy controls. This finding is consistent with the concept that whereas the prefrontal cortex is smaller in schizophrenia, it is mostly preserved in SPD. Similarly, our group reported that schizophrenia patients showed reduced gray matter volume widely across the prefrontal cortex, whereas SPD patients had reductions that did not significantly differ from those of the healthy controls and were 
Table 1 MRI results in patients with schizotypal personality disorder

Study Year SPD patients/ Findings

\begin{tabular}{llll}
\hline Dickey et al. [33] & 2002 & $21 / 22$ \\
& & \\
Dickey et al. [32] & 2003 & $21 / 29$ \\
Dickey et al. [28] & 1999 & $14 / 14$ \\
& & \\
Dickey et al. [34] & 2007 & $20 / 29$ \\
& & \\
Downhill et al. [29] & 2001 & $13 / 31(27 \mathrm{Sz})$
\end{tabular}

Goldstein et al. [22] 2009 27/45 (52 BPD)

$\downarrow$ Gray matter in left Heschl's gyrus

Found no significant differences in planum temporale

Found no significant differences in superior temporal gyrus volume

$\downarrow$ Gray matter volume in left superior temporal gyrus

Found no significant differences in volume in the amygdala, hippocampus, or parahippocampus

$\downarrow$ Volume in left and right hippocampi

$\uparrow$ Cavum septi pellucidi

$\downarrow$ Gray matter volume in temporal lobe of both patient groups

Found no significant differences in total temporal lobe volume among the 3 groups

Found no significant differences in volume of superior temporal gyrus

$\downarrow$ Volume in superior temporal gyrus compared with HCs and BPD patients

$\uparrow$ Volume in BA42 of BPD patients compared with controls

Found no significant differences in volume of BA42 between SPD patients and controls

SPD was intermediate between controls and BPD patients for BA42 volume

Hazlett et al. [18•] $2008 \quad 79 / 148(57 \mathrm{Sz}) \quad$ - Cingulate gyrus

$\downarrow$ Total gray matter volume in both SPD and Sz

$\uparrow$ Total white matter volume in both SPD and Sz

$\downarrow$ Gray matter volume and $\uparrow$ white matter volume in BA23, BA24, and BA31

$\uparrow$ White matter volume in BA24 in SPD compared with Sz (the only area in which the patient groups differed)

Found no significant differences between patient groups in total volume

- Anterior, orbital, and dorsolateral prefrontal cortex

$\uparrow$ Volume in BA10

$\downarrow$ Prefrontal volume in right hemisphere in both SPD and Sz

$\downarrow$ Volume in left hemisphere in Sz, specifically in anterior prefrontal region

$\downarrow$ Volume in BA10 in Sz compared with controls

$\downarrow$ Total gray matter volume in prefrontal cortex in $\mathrm{Sz}$

- Temporal lobe (BA22, BA21, BA20)

$\mathrm{HC}>\mathrm{SPD}>\mathrm{Sz}$ for mean temporal lobe volume as well as in left hemisphere

$\mathrm{HC}>\mathrm{SPD}>\mathrm{Sz}$ for gray matter volume

$\mathrm{Sz}>\mathrm{SPD}>\mathrm{HC}$ for white matter volume

$\downarrow$ Gray matter volume in middle temporal gyrus for both SPD and Sz

$\downarrow$ Left, but not right, volume in middle temporal gyrus for both SPD and Sz

$\downarrow$ Volume in right hemisphere for $\mathrm{Sz}$

$\downarrow$ Gray matter volume in superior and inferior temporal gyrus in $\mathrm{Sz}$

$\uparrow$ White matter volume in superior and inferior temporal gyrus in $\mathrm{Sz}$

$\downarrow$ Superior temporal gyrus volume in Sz compared with controls

Haznedar et al. [21] 2004 13/32 (27 Sz)

Found no significant differences in cingulate gyrus volume between SPD and HCs

$\uparrow$ Overall brain volume in SPD compared with Sz, but not controls

$\downarrow$ Left cingulate gyrus, specifically BA24, in Sz compared with controls

$\downarrow$ Total volume in left anterior cingulate gyrus in $\mathrm{Sz}$

Kawasaki et al. [15] 2004 25/50 (25 Sz)

$\downarrow$ Gray matter voxels in inferior frontal gyrus, insula, anterior part of superior temporal gyrus, and medial temporal region

$\downarrow$ Gray matter volume in bilateral medial frontal cortex (anterior cingulate cortex, interior frontal gyrus, medial temporal region, septal region) in $\mathrm{Sz}$

$\downarrow$ Gray matter volume in left middle frontal gyrus, orbitofrontal cortex, insula, superior temporal gyrus (planum temporale, right inferior frontal gyrus) in $\mathrm{Sz}$

$\uparrow$ Gray matter volume in left basal ganglia in $\mathrm{Sz}$ 
Table 1 (continued)

\begin{tabular}{lll}
\hline Study & Year & $\begin{array}{l}\text { SPD patients/ } \\
\text { HCs, } n\end{array}$ \\
\hline Raine et al. [16] & $2002 \begin{array}{l}\text { 16 Sz spectrum } \\
\text { group/26 } \\
\text { psychiatric } \\
\text { controls/27 }\end{array}$ \\
& & $\begin{array}{l}\text { HCs } \\
\text { Suzuki et al. [17] }\end{array}$ \\
& $2005 / 59(53 \mathrm{Sz})$
\end{tabular}

Findings

Suzuki et al. [17]

$\downarrow$ Prefrontal gray matter volume in spectrum group compared with other 2 groups

$\downarrow$ Prefrontal/whole brain volume in spectrum group compared with other 2 groups

$\downarrow$ Volume in amygdala in SPD compared with other 2 groups

$\downarrow$ Volume in hippocampus in SPD compared with other 2 groups

$\downarrow$ Straight gyrus volume in both SPD and Sz

$\uparrow$ Prefrontal gray matter volume in right hemisphere in SPD compared with other 2 groups

$\downarrow$ Volume of whole cerebral gray matter in Sz compared with other 2 groups

$\downarrow$ Total prefrontal gray matter volume in Sz compared with other 2 groups

$\downarrow$ Superior frontal gray matter volume in Sz compared with other 2 groups

$\downarrow$ Dorsal medial prefrontal cortex in Sz compared with other 2 groups

$\downarrow$ Supplementary motor cortex in Sz compared with controls

Takahashi et al. [20] $2002 \quad 24 / 48$ (40 Sz)

$\uparrow$ Intracranial volume in males

$\uparrow$ Right hemisphere white matter in both groups

$\uparrow$ Left hemisphere gray matter volume in males for both Sz and HCs

$\uparrow$ Right anterior cingulate gyrus gray and white matter in female HCs

$\downarrow$ Right anterior cingulate gyrus gray matter in female Sz compared with female HCs

$\mathrm{HCs}>\mathrm{SPD}>\mathrm{Sz}$ for right anterior cingulate gyrus gray matter volume in both males and females

Takahashi et al. [30] 2006 39/72 (65 Sz)

$\downarrow$ Volume in superior temporal gyrus in both SPD and Sz

$\uparrow$ Planum polare in right hemisphere in all groups

$\downarrow$ Volume in left planum temporale in both SPD and Sz

$\uparrow$ Left planum temporale than right in all groups

$\downarrow$ Left planum temporale in males with SPD compared with controls

$\downarrow$ Caudal superior temporal gyrus in both SPD and Sz

$\downarrow$ Left caudal superior temporal gyrus in both SPD and Sz

$\uparrow$ Temporal pole gray matter volume in female SPD compared with female Sz patients

$\downarrow$ Heschl's gyrus in Sz compared with controls

Takahashi et al. [35] 2006 39/72 (65 Sz) - Fusiform gyrus

$\downarrow$ Total FG in Sz compared with controls

$\downarrow$ Anterior FG in Sz compared with controls

$\downarrow$ Posterior FG in both SPD and Sz

- Parahippocampal gyrus

$\uparrow$ Parahippocampal gyrus in left hemisphere for all groups

- Middle temporal gyrus

$\uparrow$ Middle temporal gyrus in right hemisphere for all groups

- Inferior temporal gyrus

$\uparrow$ Inferior temporal gyrus in left hemisphere for all groups

Takahashi et al. [31] $2010 \quad 13 / 20(18 \mathrm{Sz})$

$\downarrow$ Volume in planum temporale in both SPD and Sz

$\downarrow$ Volume in caudal superior temporal gyrus in both SPD and Sz

$\downarrow$ Right caudal superior temporal gyrus in Sz compared with controls

Found no significant differences in planum polare, Heschl's gyrus, or rostral superior temporal gyrus

$B A$ Brodmann area; $B P D$ borderline personality disorder; $F G$ fusiform gyrus; $H C$ healthy control; $S P D$ schizotypal personality disorder; $S z$ schizophrenia 
Table 2 Diffusion tensor imaging results in patients with schizotypal personality disorder

\begin{tabular}{llll}
\hline Study & Year & $\begin{array}{l}\text { Schizotypal personality disorder } \\
\text { patients/healthy controls, } n\end{array}$ & Findings \\
\hline Gurrera et al. [59] & 2007 & $11 / 8$ & $\begin{array}{l}\downarrow \text { Mean left and right FA in uncinate fasciculus of patients } \\
\text { Found no significant differences in mean diffusivity } \\
\downarrow \text { FA in BA31 and BA23 of cingulum in patients }\end{array}$ \\
Hazlett et al. [60] & 2011 & $30 / 35$ & $\begin{array}{l}\uparrow \text { FA in BA25 in patients } \\
\downarrow \text { Overall FA in right hemisphere of cingulum in patients } \\
\end{array}$ \\
& & & $\begin{array}{l}\downarrow \text { Left temporal lobe FA in patients } \\
\text { Found no significant differences in frontal lobe FA in patients } \\
\text { Found no significant group differences in FA in the uncinate } \\
\text { fasciculus or cingulum bundle }\end{array}$ \\
\hline
\end{tabular}

$B A$ Brodmann area; $F A$ fractional anisotropy

about half those observed in schizophrenia [18•]. Furthermore, the schizophrenia patients showed marked reductions in the volume of Brodmann area (BA) 10 of the prefrontal cortex, while the SPD group showed greater-than-normal volume.

To our knowledge, three MRI studies in SPD have examined the cingulate gyrus, a region that is part of the limbic system and involved in emotion and attention processing [19]. Takahashi and colleagues [20] reported that compared with healthy controls, the anterior cingulate gray matter volume was significantly reduced in schizophrenia patients, but not SPD patients. Moreover, the SPD patients did not differ from the healthy controls or schizophrenia patients, indicating that they were intermediate in terms of their anterior cingulate volume. However, the SPD patients failed to show the normal pattern of $\mathrm{R}>\mathrm{L}$ asymmetry of the anterior cingulate gray and white matter. In two separate samples, our group reported no differences in cingulate gyrus volume between individuals with SPD and healthy controls $[21,22]$. However, a third study with a much larger sample of individuals with SPD (ie, $n=79$ in Hazlett et al. [18 ] vs $n=13$ in Haznedar et al. [21] and $n=$ 27 in Goldstein et al. [22]) reported smaller gray and larger white matter volume in the anterior cingulate (BA24, averaged across left and right hemisphere) compared with healthy controls $(n=148)$. It is important to note that while compared with controls, the SPD patients in Hazlett et al. [18 $]$ showed greater gray matter reductions in BA24 than the schizophrenia patients, they also had significantly greater white matter volume in this same region compared with controls and schizophrenia patients. Thus, the net volume reduction (averaged across gray and white matter) for BA24 of the anterior cingulate was greater for schizophrenia than for SPD patients. This alludes to the importance of studying large samples and also the importance of white matter, which is reviewed in the section below on DTI.
We also wish to briefly review MRI studies in SPD that examined key regions that are related to the frontal lobe. Work from our group found that the size of the pulvinar, a subcomponent of the thalamus that projects to temporal association and sensory cortices, was reduced in individuals with SPD, as well as schizophrenia patients, while the volume of the mediodorsal nucleus of the thalamus, associated with the prefrontal cortex, was decreased only in the schizophrenia patients [23]. Thus, reductions in the volume of subcortical nuclei relaying from the thalamus to cortex seem to parallel deficits in associated cortical regions in SPD (ie, temporal, but not frontal lobe abnormalities). A study examining the anterior limb of the internal capsule (ALIC) - an important frontal-striatal white matter tractreported that compared with healthy controls, individuals with SPD had significantly decreased volume in the right, but not the left ALIC [24]. Studies (e.g., Zhou et al. [25]) examining patients with schizophrenia, however, have found bilateral volume reductions in the ALIC. These findings are consistent with the concept of a spectrum of region, with SPD having an abnormality in the pulvinar while schizophrenia patients show abnormalities in two subregions of the thalamus (pulvinar and mediodorsal nucleus), and at the same time, a spectrum of severity, in which the abnormality in the ALIC is more severe in schizophrenia than observed in SPD.

\section{Temporal Lobe Findings}

Based on robust findings of smaller left superior temporal gyrus (STG) volume in schizophrenia (reviewed by Shenton et al. [11••, 26] and Levitt et al. [27]), early MRI studies in SPD examined this same region that is important for language processing. Beginning with Dickey et al. [28], several studies have reported reduced STG volume in SPD (Downhill et al. [29], Goldstein et al. [22], Kawasaki et al. [15], Takahashi et al. [30, 31]). However, Dickey et al. [28] 
found reduced STG volume in men, but later reported no reduction in women with SPD [32]. A longitudinal MRI study reported no difference in STG volume reduction over 2.7 years between the SPD and healthy control groups, whereas schizophrenia patients showed greater reduction compared with both groups [31]. Left Heschl's gyrus, a subregion of the STG, was reported to be reduced in volume in SPD [33]. Interestingly, Dickey et al. [33] found that neither right Heschl's gyrus nor the planum temporale, also a component of the STG, differed between the SPD and healthy control groups. Reduced volume of the middle [18• 29] and inferior temporal gyrus [29] has also been reported in SPD. Studies examining medial temporal lobe structures in SPD have reported reduced left/right asymmetry in the parahippocampal region [28] and reduced volume of the amygdala [17] and hippocampus $[17,34]$. A voxel-based morphometry study in SPD reported decreased gray matter in insula and entorhinal regions but no differences in the STG [14]. Takahashi et al. [35] reported reduced posterior, but not anterior, fusiform gyrus volume in SPD. However, widespread reductions in the fusiform, including both the anterior and posterior regions, were observed in the schizophrenia patients compared with the healthy controls. It is important to note that the finding of STG volume reduction in SPD was not observed in individuals with borderline personality disorder [22], suggesting that STG abnormalities may be an important endophenotype for schizophrenia spectrum disorders.

\section{Diffusion Tensor Imaging Studies in Schizotypal Personality Disorder}

\section{What is Diffusion Tensor Imaging?}

DTI is a relatively new brain imaging tool-developed in the 1990s - that has provided indirect in vivo evidence for disruptions in the coherence and direction of white matter fiber tracts [36-38]. Briefly, DTI is based on the selfdiffusion properties of water [39]. DTI tells us how much randomly diffusing water molecules prefer to go in one direction as opposed to all directions, and from this information, we can learn about brain tissues that contain water molecules [40]. Isotropic diffusion refers to the property of water molecules diffusing freely, unrestricted in any direction. However, when boundaries exist, they limit the diffusion of water molecules in certain directions, and the diffusion properties of water change to what is termed anisotropic diffusion. Axons, which have a cylindrical morphology, allow for greater diffusion along the length of the axon, and the cell membrane hinders diffusion [41]. Thus, axonal fiber tracts provide a mechanism for anisotropic diffusion. Fractional anisotropy (FA) and mean diffusivity (MD) are the two most common quantitative scalar measures used in DTI studies. MD provides a measure of the mean molecular diffusion, or diffusivity. This value provides a measure of the barriers to free diffusion in the volume, but it does not provide information about the direction of movement. FA is a measure of the degree to which diffusion is directionally constrained. This scalar measure ranges from 0 to 1 , where 0 represents no preferred direction (isotropic diffusion) and 1 represents unidirectional movement (anisotropic diffusion). These values, FA and MD, are interpreted as indicators of the integrity of white matter. In damaged white matter, MD values are higher as a result of increased free diffusion. Lower FA values reflect a loss of coherence in the preferred direction of movement. For a good review of DTI in psychiatric disorders, see White et al. [42].

\section{Diffusion Tensor Imaging in Schizophrenia}

Given the ability of DTI to measure the microstructural integrity of neuronal tracts and the considerable work implicating fronto-temporal connectivity dysfunction in schizophrenia (e.g., Akbarian et al. [43], Friston and Frith [44], Kraepelin [1], Meyer-Lindenberg et al. [45], and Weinberger et al. [46]), there have been many schizophrenia-related DTI studies. Although inconsistencies exist, the vast majority of these studies report lower FA in the frontal and temporal regions of patients with schizophrenia [47•, 48-56] (also see Kanaan et al. [57] and White et al. [42] for reviews). The meta-analysis of Ellison-Wright and Bullmore [47•, p. 3] concluded that across 15 studies, "significant reductions were present in two regions: the left frontal deep white matter and the left temporal deep white matter." As discussed previously, individuals with SPD have many similarities with schizophrenia patients, including shared genetics and phenomenology [5]. In this section, we review findings from the three DTI studies that have examined white matter integrity in SPD to date. Where possible, we also report any SPD symptom correlations with DTI measures.

\section{Diffusion Tensor Imaging in Schizotypal Personality Disorder}

The first study to examine DTI in SPD was conducted by Nakamura and coworkers [58], who examined a community sample of 15 men with SPD and 15 healthy control participants and reported lower FA in the uncinate fasciculus but found no SPD-healthy control differences in the cingulum bundle. Both of these prominent fiber bundles connect regions of the frontal and temporal lobes. Nakamura et al. [58] also reported that reduced FA in the uncinate was 
associated with greater severity of symptoms, including ideas of reference, suspiciousness, and interpersonal deficits. A pilot study examined FA in the uncinate fasciculus in 11 neuroleptic-naive men with SPD and 8 healthy men [59] and replicated the Nakamura et al. [58] finding of lower FA in SPD patients. We recently examined FA in white matter underlying BAs and reported lower FA in the left temporal lobe and right cingulum regions in a community sample of 30 SPDs compared with 35 matched healthy controls [60]. Among the individuals with SPD, lower FA in the cingulum was associated with more severe negative symptoms (e.g., odd speech).

\section{Conclusions}

\section{MRI}

Consistent with three earlier reviews of MRI imaging in SPD $[5,10,61]$, this review concurs that abnormalities in volume of the temporal lobe, particularly the STG, are consistently observed. In contrast, findings in medial temporal lobe and frontal lobe regions are more conflicting. This pattern of results is in line with the concept of a spectrum of region given that schizophrenia patients show reduced volume in both frontal and temporal regions, while individuals with SPD show reduced volume most consistently in only the temporal lobe. At the same time, frontal lobe findings in SPD are also consistent with a spectrum of compensation pattern, given that some studies have shown that patients have larger-than-normal prefrontal cortex [17] and anterior cingulum white matter [18•] volume. Thus, larger frontal lobe volume in SPD may serve to protect these individuals from the full-blown psychosis seen in schizophrenia. In sum, this variety of patterns is consistent with a multiple-gene model in which several deficits produce schizophrenia, fewer deficits produce SPD, and some protective or modulatory factors ameliorate full development of schizophrenia in SPD. This model originated from the hypothesis that schizophrenia results from the cumulative impact of multiple common small-effect, genetic variants interacting with environmental exposures to exceed a biological threshold [62].

\section{Diffusion Tensor Imaging}

It is difficult to draw a firm conclusion about DTI abnormalities in SPD given the availability of only three studies with relatively small sample sizes. Nevertheless, they consistently indicate reduced FA in the uncinate fasciculus and temporal lobe in SPD. The uncinate fasciculus is a fiber bundle that connects the inferior frontal and anterior temporal lobe. Therefore, these findings support the idea that SPD shares with schizophrenia an abnormality in frontal-temporal connectivity. The correlations with symptom severity reported by Nakamura et al. [58] further suggest that this disconnectivity may play a critical role in the cognitive distortion observed in SPD. The finding of reduced FA in the cingulum is more mixed across studies, suggesting that it may not be as robust a finding.

FA abnormalities in the uncinate fasciculus, temporal lobes, and cingulum have been reported in schizophrenia patients (e.g., Fujiwara et al. [63, 64], Hubl et al. [65], Minami et al. [66], Mitelman et al. [67], Kubicki et al. [48, 68], Burns et al. [69], Schneiderman et al. [70]). Thus, SPD shares with schizophrenia a similarity in reduced FA in the uncinate fasciculus, yet the inconsistent SPD finding of reduced cingulum FA suggests a region in which SPD and schizophrenia may differ. Consistent with the concept of frontal-lobe sparing in SPD acting as a buffer that may help minimize abnormalities of the temporal lobe $[5,18 \bullet]$, it may be that unlike schizophrenia, the cingulum is spared in SPD. In conclusion, the DTI findings to date in SPD are most consistent with the concept of spectrum of region, suggesting that schizophrenia is characterized by disconnectivity in both frontal and temporal white matter tracts, whereas in SPD, only the temporal regions show consistent abnormalities.

\section{Future Directions}

Future work using MRI in schizophrenia-spectrum disorders would benefit from multimodal measures examining both volume and FA on a within-subjects basis. For example, it is unclear what the relationship is between schizophrenia-spectrum abnormalities in the volume of white matter tracts (e.g., the anterior limb of the internal capsule and FA values within the tract). Are smaller-sized tracts associated with higher or lower FA? Also, we need to better understand the cognitive correlates of volume (both gray and white matter) and FA abnormalities across the spectrum. Additional studies that directly compare SPD and schizophrenia are sorely needed in order to better understand how these schizophrenia-spectrum groups both differ and show similarities in terms of gray and white matter abnormalities. Finally, integrating structural MRI and DTI data with genetic data will advance our understanding of the genetic contributions to structural and functional deficits. Combining these methodologies is likely to advance our understanding of the pathophysiology of schizophrenia and shed light on potential protective factors observed in SPD, which in turn may improve treatment and prognosis.

Acknowledgment Support for this work came from VA MERIT I01 CX000261 (to Dr. Hazlett). 
Disclosure No potential conflicts of interest relevant to this article were reported.

Open Access This article is distributed under the terms of the Creative Commons Attribution Noncommercial License which permits any noncommercial use, distribution, and reproduction in any medium, provided the original author(s) and source are credited.

\section{References}

Papers of particular interest, published recently, have been highlighted as:

- Of importance

•- Of major importance

1. Kraepelin E. Manic-depressive insanity and paranoia. Edinburgh: E. and S. Livingstone; 1919.

2. Bleuler E. Dementia praecox or the group of schizophrenias. New York: International Universities Press; 1950.

3. Kety SS, Rosenthal D, Wender PH, et al. Mental illness in the biological and adoptive families of adopted schizophrenics. Am J Psychiatry. 1971;128(3):302-6.

4. Rosenthal D, Wender PH, Kety SS, et al. The adopted-away offspring of schizophrenics. Am J Psychiatry. 1971;128(3):307-11.

5. Siever LJ, Davis KL. The pathophysiology of schizophrenia disorders: perspectives from the spectrum. Am J Psychiatry. 2004;161(3):398-413.

6. American-Psychiatric-Association. DSM-IV: diagnostic and statistical manual of mental disorders. 4th ed. Washington, D.C.: APA; 1994.

7. Bergman AJ, Harvey PD, Mitropoulou V, et al. The factor structure of schizotypal symptoms in a clinical population. Schizophr Bull. 1996;22(3):501-9.

8. Raine A, Reynolds C, Lencz T, et al. Cognitive-perceptual, interpersonal, and disorganized features of schizotypal personality. Schizophr Bull. 1994;20(1):191-201

9. Chakos M, Lieberman J, Bilder R. Increase in caudate nuclei volume of first episode schizophrenia patients taking antipsychotic drugs. Am J Psychiatry. 1994;151:1430-6.

10. Dickey CC, McCarley RW, Shenton ME. The brain in schizotypal personality disorder: a review of structural MRI and CT findings. Harv Rev Psychiatry. 2002;10(1):1-15.

11. •• Shenton ME, Whitford TJ, Kubicki M. Structural neuroimaging in schizophrenia: from methods to insights to treatments. Dialogues Clin Neurosci. 2010;12(3):317-32. This paper provides an excellent review and integration of neuroimaging studies in schizophrenia.

12. Eisenberg DP, Berman KF. Executive function, neural circuitry, and genetic mechanisms in schizophrenia. Neuropsychopharmacology. 2010;35(1):258-77.

13. Sanz JH, Karlsgodt KH, Bearden CE, et al. Symptomatic and functional correlates of regional brain physiology during working memory processing in patients with recent onset schizophrenia. Psychiatry Res. 2009;173(3):177-82.

14. Yoneyama E, Matsui M, Kawasaki Y, et al. Gray matter features of schizotypal disorder patients exhibiting the schizophreniarelated code types of the Minnesota Multiphasic Personality Inventory. Acta Psychiatr Scand. 2003;108(5):333-40.

15. Kawasaki Y, Suzuki M, Nohara S, et al. Structural brain differences in patients with schizophrenia and schizotypal disorder demonstrated by voxel-based morphometry. Eur Arch Psychiatry Clin Neurosci. 2004;254(6):406-14.

16. Raine A, Lencz T, Yaralian P, et al. Prefrontal structural and functional deficits in schizotypal personality disorder. Schizophr Bull. 2002;28(3):501-13.

17. Suzuki M, Zhou SY, Takahashi T, et al. Differential contributions of prefrontal and temporolimbic pathology to mechanisms of psychosis. Brain. 2005;128(Pt 9):2109-22.

18. - Hazlett EA, Buchsbaum MS, Haznedar MM, et al. Cortical gray and white matter volume in unmedicated schizotypal and schizophrenia patients. Schizophr Res. 2008;101:111-23. This paper examined volume of frontal and temporal lobe regions across the schizophrenia spectrum in a large sample of healthy controls, individuals with schizotypal personality disorder, and schizophrenia.

19. Devinsky O, Morrell MJ, Vogt BA. Contributions of anterior cingulate cortex to behaviour. Brain. 1995;118(Pt 1):279-306.

20. Takahashi T, Suzuki M, Kawasaki Y, et al. Volumetric magnetic resonance imaging study of the anterior cingulate gyrus in schizotypal disorder. Eur Arch Psychiatry Clin Neurosci. 2002;252(6):268-77.

21. Haznedar MM, Buchsbaum MS, Hazlett EA, et al. Cingulate gyrus volume and metabolism in the schizophrenia spectrum. Schizophr Res. 2004;71(2-3):249-62.

22. Goldstein KE, Hazlett EA, New AS, et al. Smaller superior temporal gyrus volume specificity in schizotypal personality disorder. Schizophr Res. 2009;112(1-3):14-23.

23. Byne W, Buchsbaum MS, Kemether E, et al. Magnetic resonance imaging of the thalamic mediodorsal nucleus and pulvinar in schizophrenia and schizotypal personality disorder. Arch Gen Psychiatry. 2001;58(2):133-40.

24. Suzuki M, Zhou SY, Hagino H, et al. Volume reduction of the right anterior limb of the internal capsule in patients with schizotypal disorder. Psychiatry Res. 2004;130(3):213-25.

25. Zhou SY, Suzuki M, Hagino H, et al. Decreased volume and increased asymmetry of the anterior limb of the internal capsule in patients with schizophrenia. Biol Psychiatry. 2003;54(4):427-36.

26. Shenton ME, Dickey CC, Frumin M, et al. A review of MRI findings in schizophrenia. Schizophr Res. 2001;49(1-2):1-52.

27. Levitt JJ, Bobrow L, Lucia D, et al. A selective review of volumetric and morphometric imaging in schizophrenia. Curr Top Behav Neurosci. 2010;4:243-81.

28. Dickey CC, McCarley RW, Voglmaier MM, et al. Schizotypal personality disorder and MRI abnormalities of temporal lobe gray matter. Biol Psychiatry. 1999;45(11):1393-402.

29. Downhill JE, Buchsbaum MS, Hazlett EA, et al. Temporal lobe volume determined by magnetic resonance imaging in schizotypal personality disorder and schizophrenia. Schizophr Res. 2001;48 (2-3):187-99.

30. Takahashi T, Suzuki M, Zhou SY, et al. Morphologic alterations of the parcellated superior temporal gyrus in schizophrenia spectrum. Schizophr Res. 2006;83(2-3):131-43.

31. Takahashi T, Suzuki M, Zhou SY, et al. A follow-up MRI study of the superior temporal subregions in schizotypal disorder and first-episode schizophrenia. Schizophr Res. 2010;119(1-3):65-74.

32. Dickey CC, McCarley RW, Voglmaier MM. An MRI study of superior temporal gyrus volume in women with schizotypal personality disorder. Am J Psychiatry. 2003;160:2198-201.

33. Dickey CC, McCarley RW, Voglmaier MM, et al. Smaller left Heschl's gyrus volume in patients with schizotypal personality disorder. Am J Psychiatry. 2002;159(9):1521-7.

34. Dickey CC, McCarley RW, Xu ML, et al. MRI abnormalities of the hippocampus and cavum septi pellucidi in females with schizotypal personality disorder. Schizophr Res. 2007;89 (1-3):49-58. 
35. Takahashi T, Suzuki M, Zhou SY, et al. Temporal lobe gray matter in schizophrenia spectrum: a volumetric MRI study of the fusiform gyrus, parahippocampal gyrus, and middle and inferior temporal gyri. Schizophr Res. 2006;87(1-3):116-26.

36. Basser PJ, Mattiello J, LeBihan D. MR diffusion tensor spectroscopy and imaging. Biophys J. 1994;66(1):259-67.

37. Moseley ME, Cohen Y, Kucharczyk J, et al. Diffusion-weighted MR imaging of anisotropic water diffusion in cat central nervous system. Radiology. 1990;176(2):439-45.

38. Pierpaoli C, Jezzard P, Basser PJ, et al. Diffusion tensor MR imaging of the human brain. Radiology. 1996;201(3):637-48.

39. Basser PJ, Mattiello J, LeBihan D. Estimation of the effective selfdiffusion tensor from the NMR spin echo. J Magn Reson B. 1994;103(3):247-54.

40. Thomason ME, Thompson PM. Diffusion imaging, white matter, and psychopathology. Annu Rev Clin Psychol. 2011;7:63-85.

41. Pajevic S, Pierpaoli C. Color schemes to represent the orientation of anisotropic tissues from diffusion tensor data: application to white matter fiber tract mapping in the human brain. Magn Reson Med. 1999;42(3):526-40.

42. White T, Nelson M, Lim KO. Diffusion tensor imaging in psychiatric disorders. Top Magn Reson Imaging. 2008;19(2):97-109.

43. Akbarian S, Kim JJ, Potkin SG, et al. Maldistribution of interstitial neurons in prefrontal white matter of the brains of schizophrenic patients. Arch Gen Psychiatry. 1996;53(5):425-36.

44. Friston KJ, Frith CD. Schizophrenia: a disconnection syndrome? Clin Neurosci. 1995;3(2):89-97.

45. Meyer-Lindenberg A, Poline JB, Kohn PD, et al. Evidence for abnormal cortical functional connectivity during working memory in schizophrenia. Am J Psychiatry. 2001;158 (11):1809-17.

46. Weinberger DR, Berman KF, Suddath R, et al. Evidence of dysfunction of a prefrontal-limbic network in schizophrenia: a magnetic resonance imaging and regional cerebral blood flow study of discordant monozygotic twins. Am J Psychiatry. 1992;149(7):890-7.

47. - Ellison-Wright I, Bullmore E. Meta-analysis of diffusion tensor imaging studies in schizophrenia. Schizophr Res. 2009;108(13):3-10. This paper was the first to conduct a comprehensive review of DTI studies in schizophrenia. It provides a good review and integration of the literature.

48. Kubicki M, Westin CF, Nestor PG, et al. Cingulate fasciculus integrity disruption in schizophrenia: a magnetic resonance diffusion tensor imaging study. Biol Psychiatry. 2003;54 (11):1171-80

49. Kumra S, Ashtari M, McMeniman M, et al. Reduced frontal white matter integrity in early-onset schizophrenia: a preliminary study. Biol Psychiatry. 2004;55(12):1138-45.

50. Lim KO, Hedehus M, Moseley M, et al. Compromised white matter tract integrity in schizophrenia inferred from diffusion tensor imaging. Arch Gen Psychiatry. 1999;56(4):367-74.

51. Sun Z, Wang F, Cui L, et al. Abnormal anterior cingulum in patients with schizophrenia: a diffusion tensor imaging study. NeuroReport. 2003;14(14):1833-6.

52. Szeszko PR, Robinson DG, Ashtari M, et al. Clinical and neuropsychological correlates of white matter abnormalities in recent onset schizophrenia. Neuropsychopharmacology. 2008;33 (5):976-84
53. Tamminga CA, Vogel M, Gao X, et al. The limbic cortex in schizophrenia: focus on the anterior cingulate. Brain Res. 2000;31 (2-3):364-70.

54. Wang F, Sun Z, Cui L, et al. Anterior cingulum abnormalities in male patients with schizophrenia determined through diffusion tensor imaging. Am J Psychiatry. 2004;161(3):573-5.

55. Ashtari M, Cottone J, Ardekani BA, et al. Disruption of white matter integrity in the inferior longitudinal fasciculus in adolescents with schizophrenia as revealed by fiber tractography. Arch Gen Psychiatry. 2007;64(11):1270-80.

56. Konrad A, Winterer G. Disturbed structural connectivity in schizophrenia primary factor in pathology or epiphenomenon? Schizophr Bull. 2008;34(1):72-92.

57. Kanaan RA, Shergill SS, Barker GJ, et al. Tract-specific anisotropy measurements in diffusion tensor imaging. Psychiatry Res. 2005.

58. Nakamura M, McCarley RW, Kubicki M, et al. Fronto-temporal disconnectivity in schizotypal personality disorder: a diffusion tensor imaging study. Biol Psychiatry. 2005;58(6):468-78.

59. Gurrera RJ, Nakamura M, Kubicki M, et al. The uncinate fasciculus and extraversion in schizotypal personality disorder: a diffusion tensor imaging study. Schizophr Res. 2007;90(13):360-2.

60. Hazlett EA, Goldstein KE, Tajima-Pozo K, et al. Cingulate and temporal lobe fractional anisotropy in schizotypal personality disorder. NeuroImage. 2011;55(3):900-8.

61. Raine A. Schizotypal personality: neurodevelopmental and psychosocial trajectories. Annu Rev Clin Psychol. 2006; 2:291-326.

62. Gottesman I, Shields J, Hanson DR. Schizophrenia, the epigenetic puzzle. New York: Cambridge University Press; 1982.

63. Fujiwara H, Namiki C, Hirao K, et al. Anterior and posterior cingulum abnormalities and their association with psychopathology in schizophrenia: a diffusion tensor imaging study. Schizophr Res. 2007;95(1-3):215-22.

64. Fujiwara H, Hirao K, Namiki C, et al. Anterior cingulate pathology and social cognition in schizophrenia: a study of gray matter, white matter and sulcal morphometry. NeuroImage. 2007;36(4):1236-45.

65. Hubl D, Koenig T, Strik W, et al. Pathways that make voices: white matter changes in auditory hallucinations. Arch Gen Psychiatry. 2004;61(7):658-68.

66. Minami T, Nobuhara K, Okugawa G, et al. Diffusion tensor magnetic resonance imaging of disruption of regional white matter in schizophrenia. Neuropsychobiology. 2003;47(3):141-5.

67. Mitelman SA, Torosjan Y, Newmark RE, et al. Internal capsule, corpus callosum and long associative fibers in good and poor outcome schizophrenia: a diffusion tensor imaging survey. Schizophr Res. 2007;92(1-3):211-24.

68. Kubicki M, Westin CF, Maier SE, et al. Uncinate fasciculus findings in schizophrenia: a magnetic resonance diffusion tensor imaging study. Am J Psychiatry. 2002;159(5):813-20.

69. Burns J, Job D, Bastin ME, et al. Structural disconnectivity in schizophrenia: a diffusion tensor magnetic resonance imaging study. Br J Psychiatry. 2003;182:439-43.

70. Schneiderman JS, Buchsbaum MS, Haznedar MM, et al. Age and diffusion tensor anisotropy in adolescent and adult patients with schizophrenia. NeuroImage. 2009;45(3):662-71. 